\title{
Exciton density pattern formation in laser irradiated quantum wells under electrodes of various shapes
}

\author{
V.V. Tomylko, I.Yu. Goliney, A.A. Chernyuk, and V.I. Sugakov \\ Institute for Nuclear Research, NAS of Ukraine, 47 Nauki Ave., Kyiv 03680, Ukraine \\ E-mail: tomylko.slava@gmail.com
}

Received December 18, 2013, published online June 23, 2014

\begin{abstract}
The condensation of indirect excitons in double quantum wells is studied in an electric field created by electrodes of different shapes. The finite value of the exciton lifetime, the pumping and nonuniformity of the electric field under the electrode are taken into account. It is shown that islands of exciton condensed phase emerge under electrodes when the pumping exceeds a certain threshold value. They appear first under the rim where the potential energy of excitons has a dip. Calculations predict a complicated evolution of the exciton density distribution: from the gaseous phase at low laser intensities to the condensed phase in the whole area under the electrode at larger intensities. Therefore, the configurations of the exciton condensed phase may be manipulated by choosing the setups with conductive electrodes of different shapes via forming specific potentials of the electrical field and controlled by the level of the laser irradiation.
\end{abstract}

PACS: 71.35.Lk Collective phenomena;

73.21.Fg Quantum wells.

Keywords: quantum wells, condensed phase, indirect exciton, macroscopic trap.

\section{Introduction}

In recent years semiconductor double quantum wells remain a very popular and convenient physical system for studying the properties of indirect excitons in which an electron and a hole are separated by an electric field to different quantum wells [1]. As a consequence, the recombination of the electron and the hole is inhibited and that causes the lifetime of indirect excitons to be by several orders of magnitude higher than the direct exciton lifetime. The study of indirect excitons is promising in terms of fundamental science, because excitons can be accumulated to a high density and many-exciton effects can be investigated. Additionally, the system of indirect excitons can be promising for applications, since they can travel over large distances carrying energy and information and may be used in the double quantum well based semiconductor devices [2,3]. A number of recent papers reported emergence of the macroscopic scale spatial structuring in the emission from the systems of high density indirect excitons in the double quantum wells based on GaAs/AlGaAs. Thus, the authors [4] observed a break-down of the emitting ring outside the laser spot into separate fragments periodically localized along the ring. In the paper [5], in which the excitation of the quantum well was carried out through a window in a metallic electrode, the authors found a periodical structure of the luminescent islands situated along the ring under the perimeter of the window. The appearance of the structures in the exciton density distribution was observed for a periodical potential applied to excitons [6]. Recently Timofeev and co-authors [7,8] presented examples of emitting structures, obtained through differently shaped windows in the electrodes: a rectangle, a triangle, two circles, two triangles, etc. In order to increase the exciton concentration at the same pumping a number of works [9-12] proposed to create so-called macroscopic traps for excitons. Such traps allow accumulating excitons up to higher density without increasing the laser pumping while avoiding negative effects of heating.

Several different theoretical models of the formation of spatial patterns were proposed [13-20]. In the listed papers, the main efforts were applied for the ascertainment of the principal possibility of the onset of the periodicity in the exciton density distribution. They come short of the explanation of numerous experiments with different setups, at different pumping, temperature, for different type of external fields.

The explanation of the experimentally observed spatial structures in the exciton density distribution was given in the papers [21-25]. The theoretical approaches of these works are based on two major assumptions: 1) there exists 
an exciton condensed phase caused by an attractive interaction between excitons; 2) the finite value of the exciton lifetime plays an important role in the formation of the exciton condensed phase. The first assumption is supported by the existence of bound states of two indirect excitons, obtained in the calculations by several groups [26-28], and a new phase in the multiexciton system [29]. The attractive exchange and van der Waals interactions may exceed the repulsive long-range dipole-dipole interactions between excitons if the distance between the quantum wells in a double well setup, and, correspondingly, dipole moments of indirect excitons, are not too large. The second assumption is connected with the fact that the exciton lifetime is less than the time of the establishment of the equilibrium between different phases.

In this paper, we apply our approbated approach to new systems investigating the formation of the exciton density patterns in double quantum wells in an external electric field applied by using metal electrodes of various spatial shapes: disk-shaped, square-shaped, rectangular-shaped and triangle-shaped electrodes. The paper consists of two main parts. The first one explains the procedure of the calculations of the electrostatic field for the setups of different shapes. The second part presents the results of the calculations and analysis of the exciton density distributions for these setups. The exciton density distribution is described phenomenologically using Landau model of phase transitions generalized for particles with the finite lifetime. This approach is based on two main assumptions used in our previous papers [21-25,30,31] that were successful in the explanation of experimental results.

\section{Model of the system. Electric field potential}

We consider a double quantum well sandwiched between two flat metal electrodes. The upper electrode is finite-shaped (see Fig. 1). Let us direct the $z$ axis perpendicular to the quantum wells plane, then the $X Y$ plane will be quantum well plane. The electric potential difference

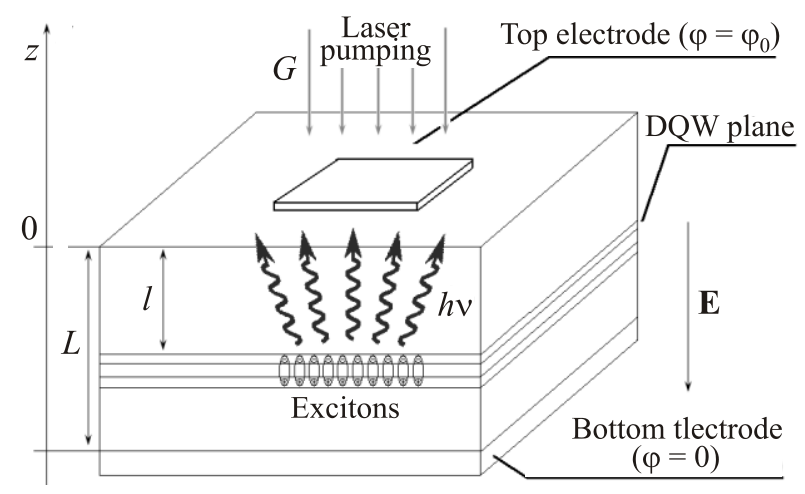

Fig. 1. Setup of the system in the case when the top electrode is square-shaped. Potentials of the top and the bottom electrodes are $\varphi_{0}$ and 0 , respectively.
$\Delta \varphi$ is applied to the electrodes, the top finite-sized electrode has a potential $\varphi_{0}$ and the bottom electrode has potential 0 . The double quantum well where excitons reside is at a distance from the top electrode. The top finite size electrode creates a nonuniform electric field. An indirect exciton, having a dipole moment, acquires an additional energy $V=-\mathbf{p E}$.

The calculation of the electric field under the electrode of an arbitrary shape is a separate problem. Analytical solution is known for a disk-shaped electrode [32]. This solution has been used in the paper [31]. Remarkably, while the electrostatic potential does not have any feature under the rim of the disk, the electric field strength does have a dip. The physical significance of this is that while neither electrons nor holes are pulled under the disk the indirect excitons, having a dipole moment, are attracted to the regions situated under the rim. Therefore an electrode of a finite size creates potential energy dips for excitons and for excitons only. The electric field under an electrode of arbitrary shape has to be calculated numerically. We have developed a simple procedure to perform these calculations and tested it for the case of the disk where the analytical solution is known. After obtaining a satisfactory approximation we have applied the same procedure to other geometries.

To find the electric field we need to know how the charges are distributed on the surface of the electrode. The electrostatic potential in the system is determined by the surface charge density $\sigma$ on the top electrode

$$
\varphi(x, y, z)=\iint_{S} \frac{\sigma\left(x^{\prime}, y^{\prime}\right)}{\sqrt{\left(x-x^{\prime}\right)^{2}+\left(y-y^{\prime}\right)^{2}+z^{2}}} d x^{\prime} d y^{\prime} .
$$

The surface charge is determined by the condition $\varphi(x, y, 0)=\varphi_{0}$ for any point on the electrode. The solution of the integral equation is difficult because of a singularity in the kernel. To avoid the difficulty we simulate the continuous charge distribution $\sigma(x, y)$ with a set of point charges $\left\{q_{i}\right\}$ situated slightly above the electrode on a grid (Fig. 2). We demand that the potentials of this system of charges were equal to $\varphi_{0}$ in the points on the surface of the electrodes below the charges. The charges $\left\{q_{i}\right\}$ can be found from the system of linear equations

$$
\left\{\sum_{i, j}^{N} \frac{q_{i j}}{\sqrt{h^{2}\left(i^{2}+j^{2}\right)+\delta^{2}}}=\varphi_{0},\right.
$$

where $h$ is the step of the grid and $\delta$ is the elevation of the grid over the electrode's plane.

To take into account influence of the bottom electrode on the electric field the method of mirror images has been used as for the similar problem in the article [31]. The proposed procedure, applicable for any flat electrode, gives solutions that while not being the solutions of the initial problem approximate these solutions satisfactory if $\delta$ is 


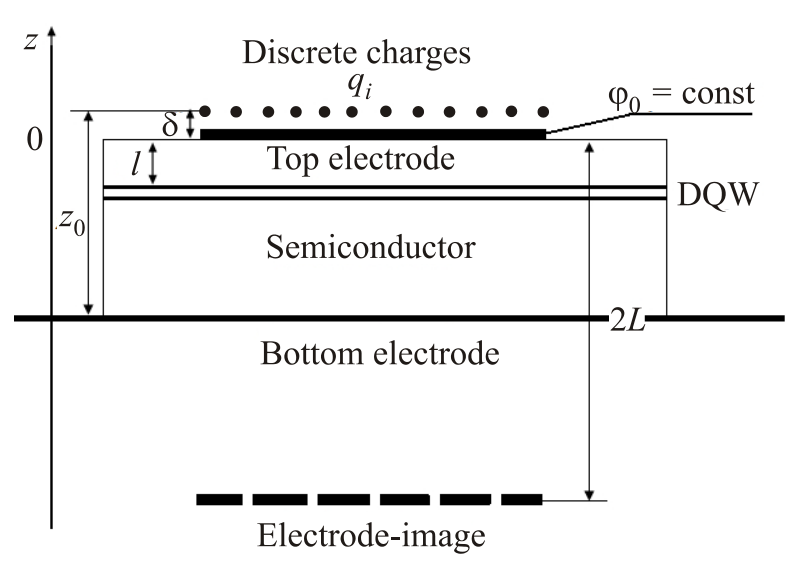

Fig. 2. Illustration of the procedure of the calculation of the electrostatic field profiles. The top metal electrode is approximated by a grid of discrete point charges. To avoid singularity charges are shifted by $\delta$ above the electrode. $l$ is the distance between the top electrode and the plane of quantum wells.

small enough compared to $h$. This has been checked for the disk-shaped electrode.

The electric potential at the level of the double quantum well is nonuniform. As in the case of the known solution for a disk, the electric field strength has a minimum directly below the rim of the electrode, especially pronounced under the corners (see Fig. 3 for the square-shaped electrode). The electric field affects the potential energy of excitons, while the potential energy of the charged particles such as electrons and holes is affected by the electrostatic potential itself which remains smooth. Therefore, the macroscopic traps appear for excitons only.

\section{Exciton density patterns}

We use the phenomenological model of phase transitions generalized for particles of the finite lifetime for simulation of the exciton density profiles under electrodes of different shapes. This section provides only a brief description of the simulation method, a detailed description of the approach can be found, for example, in [25,30]. The timedependent distribution of the exciton density $n$ satisfies the nonlinear equation:

$$
\frac{\partial n}{\partial t}=\frac{D}{k_{B} T} \nabla\left[n \nabla\left(-K \Delta n+\frac{d f}{d n}+V\right)\right]+G(\mathbf{r})-\frac{n}{\tau},
$$

where $D$ is the exciton diffusion coefficient, $k_{B}$ is the Boltzman constant, $T$ is the temperature,

$$
f(n)=f_{0}+\frac{a}{2} n^{2}+\frac{b}{3} n^{3}+\frac{c}{4} n^{4}+k_{B} T n(\ln n-1)
$$

is the free energy density, $V=V(x, y, z)$ is the external potential energy, $\tau$ is the exciton lifetime, $G(\mathbf{r})$ is the exciton creation rate due to the laser pumping, i.e., the number of excitons created in unit time per unit surface of the quantum well. $a, b, c$ are phenomenological coefficients.

Using dimensionless units for time, length, exciton concentration and energy $t_{u}=d_{1} l_{u}{ }^{2} / D, l_{u}=\sqrt{K / a}, n_{u}=$ $=\sqrt{a / c}, \quad V_{u}=a n_{u}$, respectively, where $d_{1}=k_{B} T / V_{u}$, we get a dimensionless equation for the exciton density:

$$
\frac{\partial n}{\partial t}=d_{1} \Delta n+\nabla\left[n \nabla\left(-\Delta n+n+b_{1} n^{2}+n^{3}+V\right)\right]+G(\mathbf{r})-\frac{n}{\tau}
$$

The first term describes the diffusion of excitons; the terms in brackets are due to the free energy contribution and the external potential energy. Equation (2) is a nonlinear phenomenological equation which describes the exciton density distribution taking into account the finite exciton lifetime and the external laser pumping. We have chosen the exciton creation rate in the form of a spot with the Gaussian form $G=G_{0} \exp \left[-r^{2} /\left(2 \Delta^{2}\right)\right]$, where $G_{0}$ characterizes the intensity and $\Delta$ defines the radius of a spot.

The potential $V(r)$ creates a trap for excitons. The excitons would tend to localize under the electrode, especially under the rim and the corners where the trap is deeper. With increasing pumping the processes of the conden-

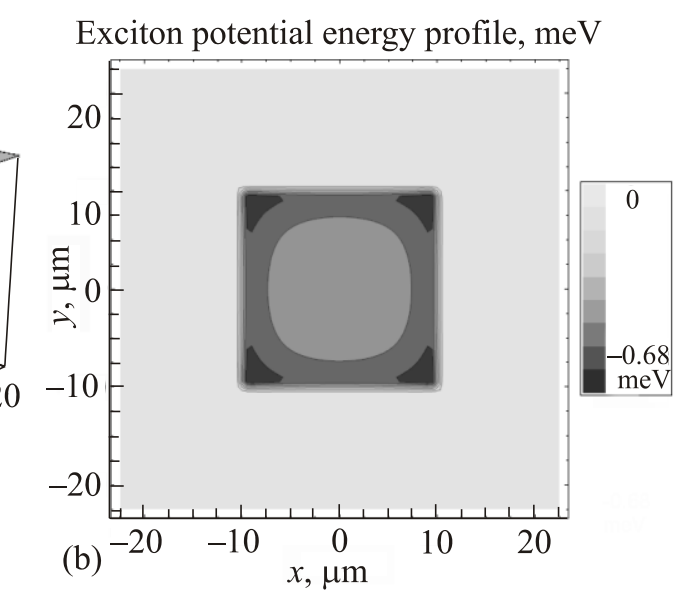

Fig. 3. 2D (a) and 3D (b) profiles of the exciton potential energy for the case of a square-shaped electrode. The applied bias is $1 \mathrm{~V}$, the electrode size is $15 \times 15 \mu \mathrm{m}$, the distance to the quantum wells plane is $l=0.2 \mu \mathrm{m}$. Potential energy depth at minimum is $0.68 \mathrm{meV}$. 
sation occur. As the result of the finite value of the exciton lifetime various patterns emerge in the density distribution. Due to the nonhomogeneous potential of the finite size electrode, the islands of the condensed phase arise at first under the rim of the electrode. Below we present the dynamics of the modification of the density distribution with increasing pumping.

Firstly, let us consider the steady-state exciton density distribution in the quantum wells plane for different spatial shapes of electrodes. Four types of finite-shaped electrodes have been used: a disk, a square, a rectangle and a triangle. The trap depth depends on the voltage applied to the electrodes and the position of the quantum wells plane relative to the top electrode. This provides a possibility to tune the configuration of the trap in a controllable manner. Examples of the steady-state solutions of the Eq. (2) for 4 setups are presented in the Fig. 4. One can see that the accumulation of excitons with the creation of the islands of the condensed phase tends to occur under the rim of the electrodes, especially under the corners where the traps are deeper.

Exciton density patterns appear only when the laser pumping exceeds a certain threshold value. The regions of the high exciton density correspond to the condensed phase and the regions of the low density correspond to the gaseous phase. The results show that the trapping of excitons takes place along the electrodes rims in all cases, inde- pendent of the electrodes' shapes. Note, that in the case of the rectangular-shape electrode the islands of the condensed phase appear not only in vicinity of the electrode's corners, but along the longer sides as well. This indicates that the condensation of excitons plays an important role in pattern formation.

The systems with the finite-size electrodes have not been realized experimentally so far. Therefore, our calculations provide predictions of the density patterns. Similar systems with differently shaped windows in the conductive electrodes have been constructed experimentally $[5,7,8]$.

Secondly, let us explore the dynamics of the exciton density pattern with increasing the laser pumping intensity for the square-shaped electrode (see Fig. 5). The detailed analysis shows complicated modification of the exciton density distribution: from the gaseous phase at low laser intensities to the condensed phase at large intensities. When the laser pumping is below a certain threshold value the exciton distribution is smooth without any particular features, almost uniform (Fig. 5(a)). As the pumping increases, the excitons start to condense, at first in the vicinity of the electrode's corners and when the pumping exceeds a certain threshold value the islands of the condensed phase emerge (Figs. 5(b), (c)). The spatial structure becomes even more complicated at higher excitation level: additional islands of the condensed phase grow between the corners along the rims of electrode (Fig. 5(d)). At still
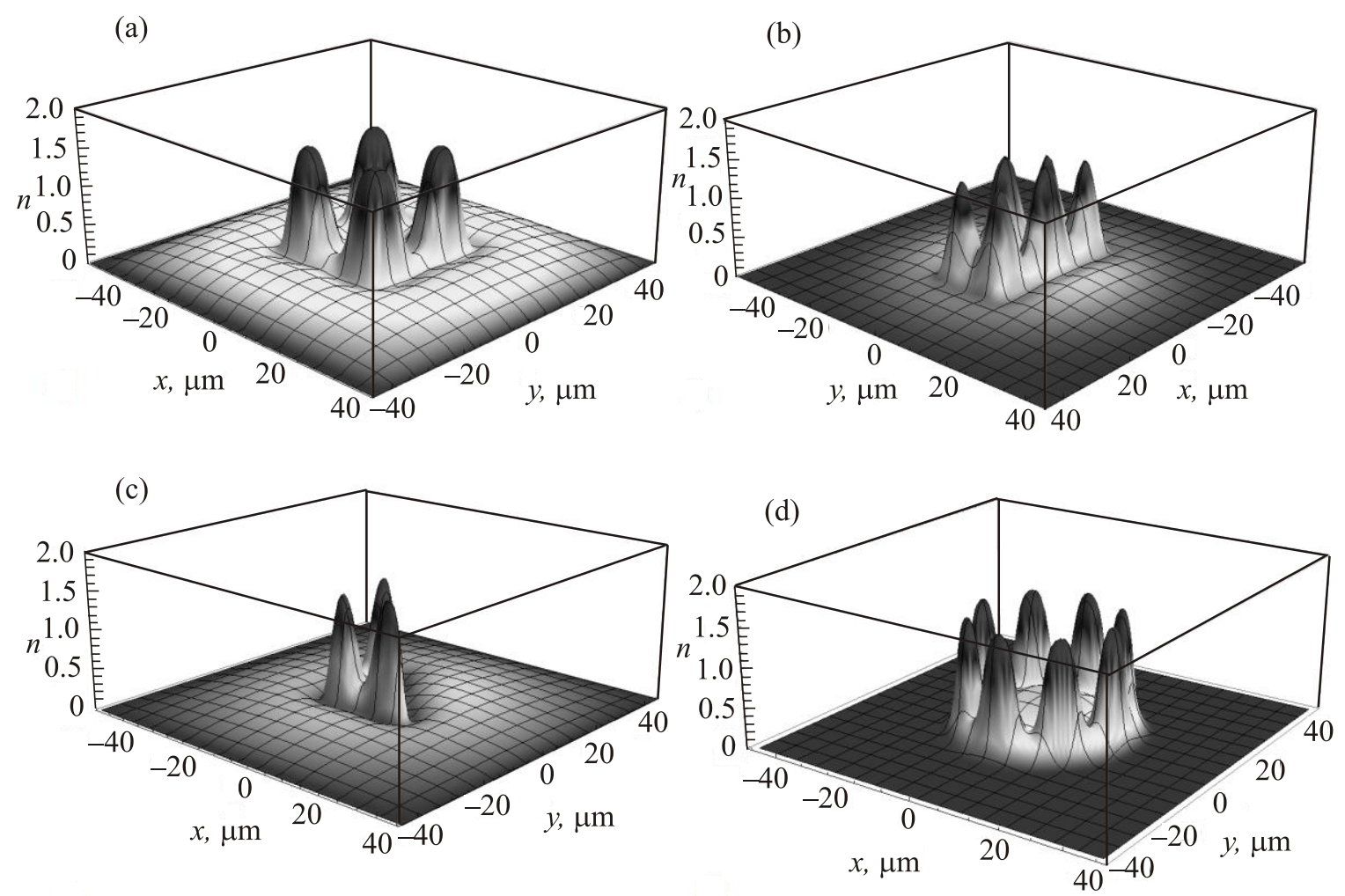

Fig. 4. Exciton density distribution in the QW plane for electrodes of different shape: (a) square $15 \times 15 \mu \mathrm{m}$; (b) rectangle $9 \times 18 \mu \mathrm{m}$; (c) triangle $7 \times 7 \times 7 \mu \mathrm{m}$; (d) disk with radius $15 \mu \mathrm{m}$. Islands of exciton condensed phase appear first in the regions under the electrodes' corners. The following parameters are used for numerical simulations: applied potential difference is $1 \mathrm{~V}$, distance to quantum wells plane is $l=0.2 \mu \mathrm{m}$. 

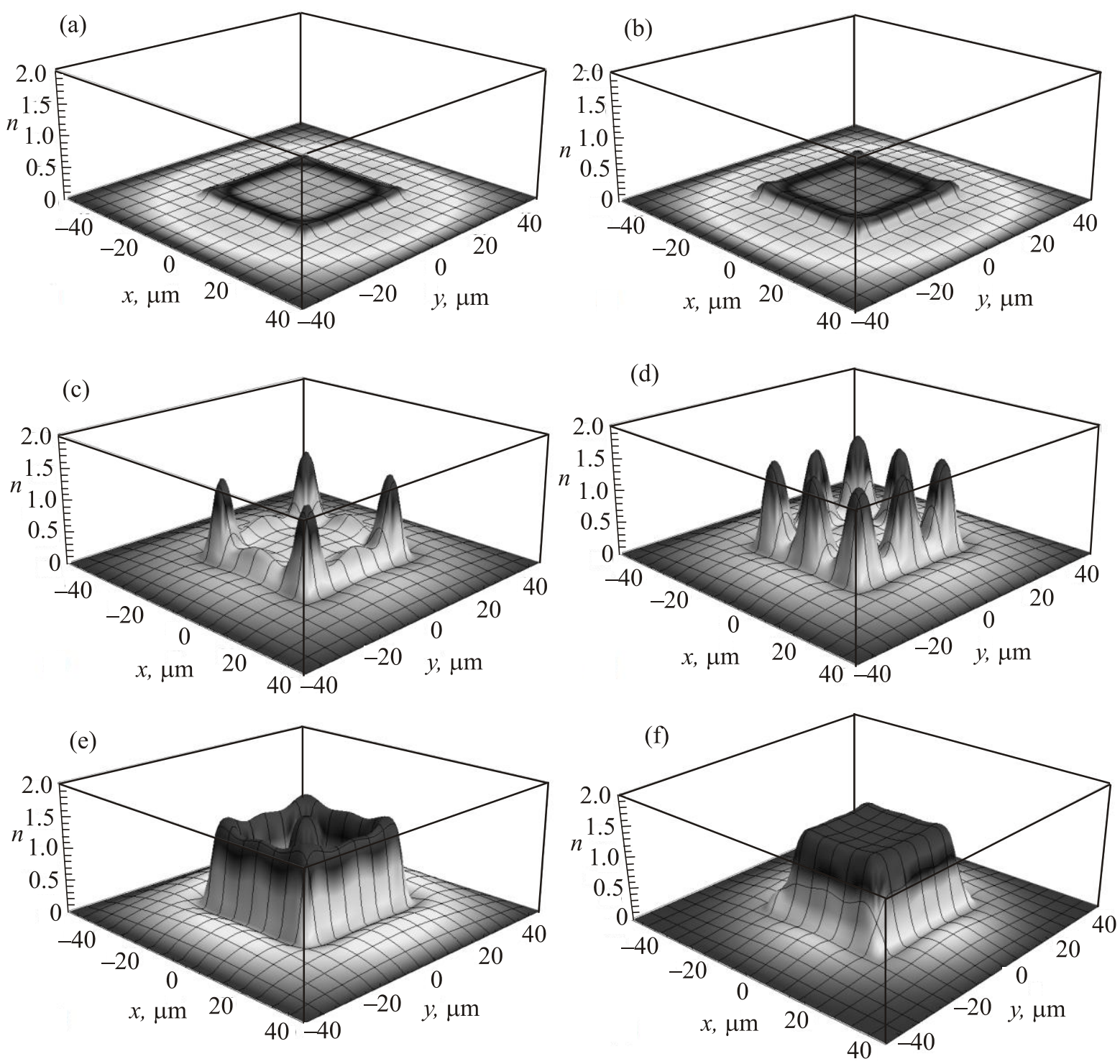

Fig. 5. Exciton density distribution in the quantum well plane for different values of the exciton creation rate characteristic $G_{0}$ in dimensionless units for the square-shaped electrode $20 \times 20 \mu \mathrm{m}: 0.001$ (a); 0.003 (b); 0.004 (c); 0.005 (d); 0.007 (e); 0.015 (f). The other parameters are the same as in Fig. 4.

stronger pumping they merge creating a continuous wall (Fig. 5(e)). And, finally, at very large intensities of the laser pumping, the condensed phase, without any inclusions of gaseous phase, occupies the whole space under the electrode (Fig. 5(f)). Such dynamics is really similar to process of phase transition saturated steam-liquid phase. Thus, the situations when only the gaseous or only the condensed phases exist are separated by the coexistence of the condensed and gaseous phases at the intermediate level of pumping.

\section{Conclusions}

In this paper we have studied formation of patterns in the exciton density distribution in laser irradiated double quantum wells with macroscopic traps for excitons. Simu- lations were performed for traps created by metal electrodes of different spatial shapes: a disk, a square, a rectangle and a triangle. Calculations have shown that the electric field created by electrodes is strongly nonuniform along its rims. The traps appear for excitons only and do not emerge either for electrons or the holes. The theory of phase transitions generalized for unstable particles has been applied for the study of the exciton density distribution. The regions of the condensed phase shaped as islands arise when the laser pumping exceeds a certain threshold value, parameters of which depend on the pumping, the lifetime of the excitons, and the structure of the external field. We report also the dynamics of the modification of the exciton density profiles with increasing the laser pumping: the condensation starts under the corners or under the rim of the electrodes where the exciton energy has minima. 
Additional condensed phase islands emerge at higher pumping at a certain distance from the minima, at still higher pumping separate islands merge into a continuous wall encircling a gaseous phase in the center of the underelectrode region with one additional island of the condensed phase, and, finally, the continuous condensed phase occupies the entire area under the electrode. The formation of patterns is caused by distinct features of phase transitions in the nonequilibrium systems of finite lifetime particles.

The considered system may useful for the study of the dynamics of phase transitions in nonhomogeneous and nonequilibrium systems and for the creation of various distributions of the exciton density controlled by the laser irradiation applicable in the optoelectronics.

1. T. Fukuzawa, E.E. Mendez, and J.M. Hong, Phys. Rev. Lett. 64, 3066 (1990).

2. G. Grosso, J. Graves, A.T. Hammack, A.A. High, L.V. Butov, M. Hanson, and A.C. Gossard, Nature Photonics 3, 577 (2009).

3. S. Lazic, A. Violante, K. Cohen, R. Hey, R. Rapaport, and P.V. Santos, arXiv:1310.6912vl [cond-mat. mes-hall] (2013).

4. L.V. Butov, A.C. Gossard, and D.S. Chemla, Nature 418, 751 (2002).

5. A.V. Gorbunov and V.B. Timofeev, JETP Lett. 83, 146 (2006).

6. M. Remeika, J.C. Graves, A.T. Hammark, A.D. Meyertholen, M.M. Fogler, L.V. Butov, M. Hanson, and A.C. Gossard, Phys. Rev. Lett. 102, 186803 (2009).

7. V.B. Timofeev, A.V. Gorbunov, and D.A. Demin, Fiz. Nizk. Temp. 37, 229 (2011) [Low Temp. Phys. 37, 179 (2011)].

8. A.V. Gorbunov and V.B. Timofeev, JETP Lett. 96, 145 (2012)

9. V. Negoita, D. W. Snoke, and K. Eberl, Appl. Phys. Lett. 75, 2059 (1999).

10. Z. Vörös, D.W. Snoke, L. Pfeiffer, and K. West, Phys. Rev. Lett. 97, 016803 (2006).

11. R. Rapaport, G. Chen, S. Simon, O. Mitrofanov, L. Pfeiffer, and P. Platzman, Phys. Rev. B 72, 075428 (2005).
12. M. Remeika, M.M. Fogler, L.V. Butov, M. Hanson, and A.C. Gossard, Appl. Phys. Lett. 100, 061103 (2012).

13. L.S. Levitov, B.D. Simons, and LV Butov, Phys. Rev. Lett. 94, 176404 (2005).

14. A.V. Paraskevov and T.V. Khabarova, Phys. Lett. A $\mathbf{3 6 8}$, 151 ( 2007).

15. R.B. Saptsov, JETP Lett. 86, 687 (2008).

16. C.S. Liu, H.G. Luo, and W.C. Wu, J. Phys.: Condens. Matter 18, 9659 (2006).

17. V.K. Mukhomorov, Phys. Solid State 52, 241 (2010).

18. J. Wilkes, E.A. Muljarov, and AL Ivanov, Phys. Rev. Lett. 109, 187402 (2012).

19. S.V. Andreev, Phys. Rev. Lett. 110, 146401 (2013).

20. V.S. Babichenko and I.Ya. Polishchuk, JETP Lett. 97, 726 (2013).

21. V.I. Sugakov, Solid State Commun. 134, 63 (2005).

22. V.I. Sugakov, Fiz. Nizk. Temp. 32, 1449 (2006) [Low Temp. Phys. 32, 1104 (2006)].

23. A.A. Chernyuk and V.I. Sugakov, Phys. Rev. B 74, 085303 (2006).

24. V.I. Sugakov and A.A. Chernyuk, JETP Lett. 85, 570 (2007).

25. V.I. Sugakov, J. Phys.: Condens. Matter 21, 275803 (2009).

26. M.Y.J. Tan, N.D. Drummond, and R.J. Needs, Phys. Rev. B 71, 033303 (2005).

27. Ch. Schindler and R. Zimmermann, Phys. Rev. B 78, 045313 (2008).

28. A.D. Meyertholen and M.M. Fogler, Phys. Rev. B, 78, 235307 (2008).

29. Yu.E. Lozovik and O.L. Berman, JETP Lett. 64, 573 (1996).

30. A.A. Chernyuk and V.I. Sugakov, Solid State Commun. 149, 2185 (2009).

31. A.A. Chernyuk, V.I. Sugakov, and V.V. Tomylko, J. Phys.: Condens. Matter 24, 195803 (2012).

32. L.D. Landau and E.M. Lifshitz, Course of Theoretical Physics (Electrodynamics of Continuous Media), Vol. 8, 2nd edition, Oxford-Pergamon (1984). 